\title{
CMEARTICLE \\ Clinics in diagnostic imaging (147)
}

Tien Jin $\underline{\operatorname{Tan}}^{1,2}$, MBBS, FRCR, Lester Chee Hao Leong ${ }^{1}$, MBBS, MMed, L/wellyn Shao-Jen $\underline{\operatorname{Sim}}^{1}$, MBBS, FRCR
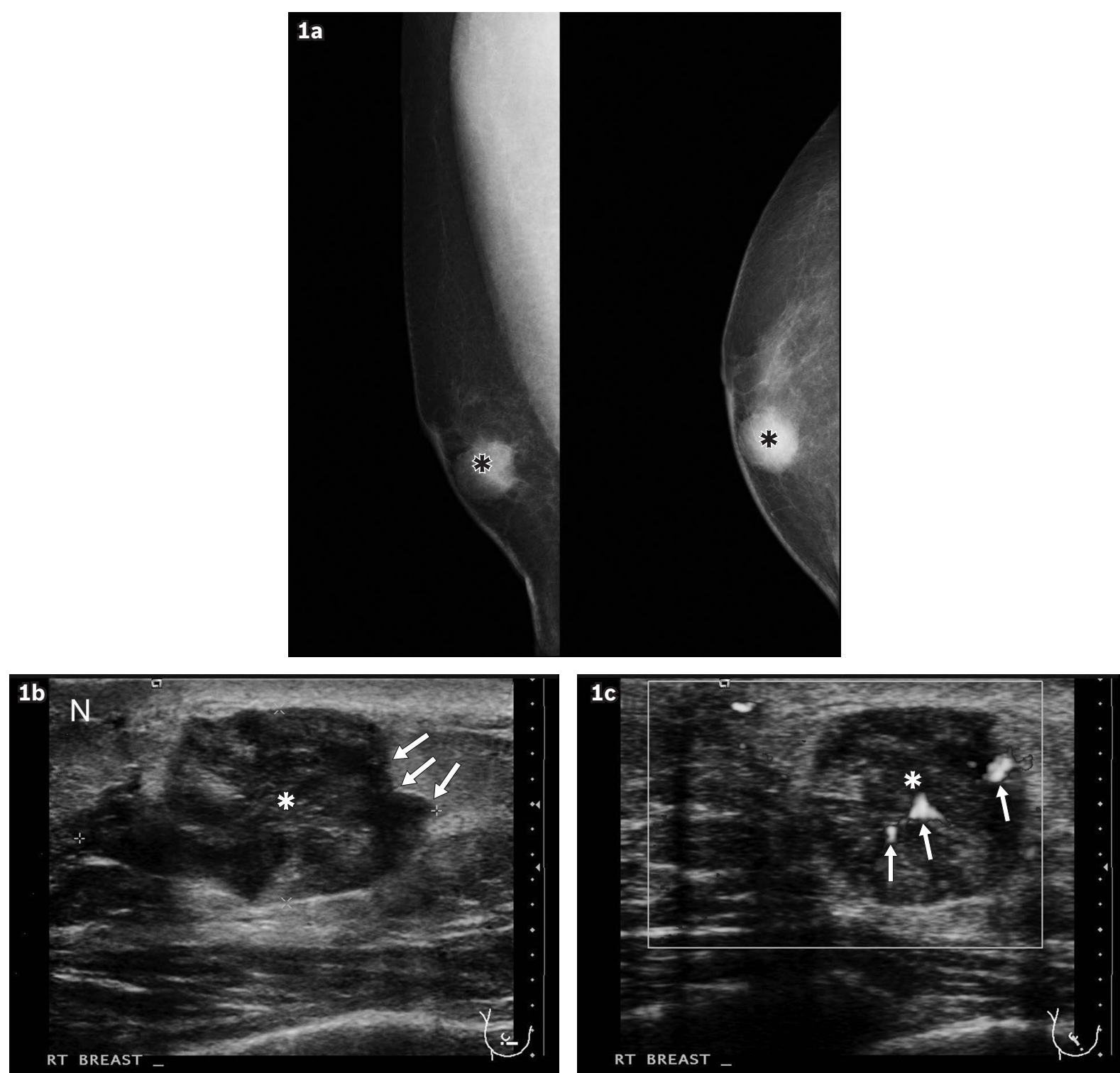

Fig. 1 (a) Mediolateral oblique and craniocaudal mammographic images of the right breast. (b \& c) Targeted US images of the clinically palpable right breast lump.

\section{CASE PRESENTATION}

A 51-year-old man with no significant medical history was referred to our institution for further management of a palpable, painless right breast lump, which had gradually increased in size for a period of six months. There had also been intermittent discharge from the right nipple for a period of two years. The discharge was bloody at onset but subsequently became clear.
Physical examination revealed a firm right breast lump with no clinical evidence of skin involvement or axillary lymphadenopathy. At the time of physical examination, the right nipple discharge was observed to be non-bloody. Mammography (Fig. 1a) and breast ultrasonography (Figs. 1b \& c) were performed. What do these images demonstrate? What is the level of suspicion for malignancy?

${ }^{1}$ Department of Diagnostic Radiology, Singapore General Hospital, ${ }^{2}$ Department of Diagnostic Radiology, Changi General Hospital, Singapore

Correspondence: Dr Tan Tien Jin, Associate Consultant, Department of Diagnostic Radiology, Changi General Hospital, 2 Simei Street 3, Singapore 529889. tien_jin_tan@cgh.com.sg 


\section{IMAGE INTERPRETATION}

Mammographic images of the right breast (Fig. 1a) demonstrate a discrete, dense, round mass with spiculated margins (black asterisks) in the periareolar lower inner quadrant of the right breast. This mass corresponds to the clinically palpable lump. No associated pathological microcalcification or axillary lymphadenopathy was evident on mammography. Corresponding targeted ultrasonography of the palpable right breast lump (Fig. 1b) confirms the presence of a heterogeneous mass (white asterisk) with irregular and angulated margins (white arrows), which is located eccentric to the nipple-areolar complex $(\mathrm{N})$. Colour Doppler imaging (Fig. 1c) of the right breast mass (white asterisk) shows the presence of increased internal vascularity (white arrows).

\section{DIAGNOSIS}

Male breast carcinoma.

\section{CLINICAL COURSE}

The patient underwent ultrasonography-guided core needle biopsy of the right breast mass, and the results confirmed the diagnosis of invasive ductal carcinoma. The tumour was positive for oestrogen and progesterone receptor, but negative for human epidermal growth factor receptor 2 . Right mastectomy and sentinel lymph node biopsy were subsequently performed, and no evidence of metastasis was found in the sentinel lymph node. The patient was commenced on adjuvant hormone therapy with tamoxifen. There was no clinical or imaging evidence of tumour recurrence one year following surgery.

\section{DISCUSSION}

Male breast carcinoma is an uncommon malignancy, accounting for only about $1 \%$ of all cases of breast cancer, except in subSaharan Africa where $7 \%-14 \%$ of breast cancers occur in men. ${ }^{(1)}$ The mean age of male breast carcinoma patients is 65 years, although this malignancy can occur at any age. ${ }^{(1)}$ Approximately $85 \%$ of breast cancers in men are moderately or poorly differentiated invasive ductal carcinoma. ${ }^{(2)}$

In male breast carcinoma, there are multiple risk factors, including high oestrogen states (e.g. Klinefelter's syndrome, exogenous oestrogen), androgen deficiency (e.g. cryptorchidism, orchidectectomy), a positive family history (which may be linked to breast cancer mutation genes BRCA1 and BRCA2 in some families), as well as prior irradiation of the thoracic wall. ${ }^{(1)}$ It has also been previously reported that up to $40 \%$ of cases of male breast carcinoma are associated with coexistent gynaecomastia, although no definite causality has been established.(2)

The most commonly encountered benign mimic of male breast carcinoma is gynaecomastia. ${ }^{(3)}$ This condition is characterised by hyperplasia of the ductal and stromal elements, mainly in the breasts of adolescent boys and men aged over
50 years. Gynaecomastia results from a state of relative oestrogen excess or androgen deficiency. Causative factors include adrenal or pituitary dysfunction, systemic disorders (e.g. hepatic cirrhosis, chronic renal failure), drugs (e.g. exogenous oestrogen, digitalis, cimetidine, spironolactone, thiazide) and neoplasms (e.g. testicular, adrenal, pituitary, lung malignancies). ${ }^{(1)}$ In some cases, no cause may be found despite extensive investigations (i.e. idiopathic gynaecomastia). Gynaecomastia is distinct from pseudogynaecomastia, a condition in which breast enlargement is due to the accumulation of fatty tissue in obese men.

There are several clinical features that may help to differentiate male breast carcinoma from gynaecomastia. ${ }^{(1)}$ Men with gynaecomastia usually present clinically with mobile and occasionally painful central subareolar masses that are either soft or firm in consistency. The mass is usually bilateral and asymmetrical, although it can also be unilateral. There is no associated axillary adenopathy or involvement of the overlying skin and nipple. Male breast carcinoma, however, typically manifests as a unilateral, painless breast mass that is either in a central subareolar location or eccentric to the nipple-areolar complex. A malignant breast mass usually has a rubbery or hard consistency, and may be fixed to the skin or underlying pectoral fascia. There may also be associated nipple retraction or discharge, skin involvement and axillary lymphadenopathy.

When gynaecomastia and male breast carcinoma are indistinguishable based on clinical findings alone, the patient should undergo mammography, which has high sensitivity, specificity and negative predictive values for distinguishing malignant from benign breast diseases. ${ }^{(4-6)}$ Breast ultrasonography is also useful for evaluating male patients who present with breast masses and nipple discharge, or when the mammographic findings are not characteristic of gynaecomastia. ${ }^{(5,6)}$

Mammographic or breast ultrasonographic primary findings of a unilateral and discrete mass in a male breast, at an eccentric location with respect to the nipple-areolar complex, should always raise the suspicion of a breast carcinoma..$^{(3,7,8)}$ This is particularly true for a solid or complex cystic breast mass that demonstrates spiculated, angulated or microlobulated margins, and increased vascularity. ${ }^{(3,7-9)}$ Pathological microcalcifications are less commonly encountered since they occur primarily in ductal carcinoma in situ components and are not frequently present in male breast carcinoma. ${ }^{(10)}$ If present, they tend to be fewer in number, coarser and less frequently rod-shaped, as compared to those seen in female breast carcinoma. The additional presence of nipple retraction or discharge, skin thickening and axillary lymphadenopathy are also important secondary features of a breast malignancy that warrants biopsy of the primary breast mass..$^{(1,3,7,8)}$

Gynaecomastia can also appear mass-like with poorly marginated borders on mammography and ultrasonography, 

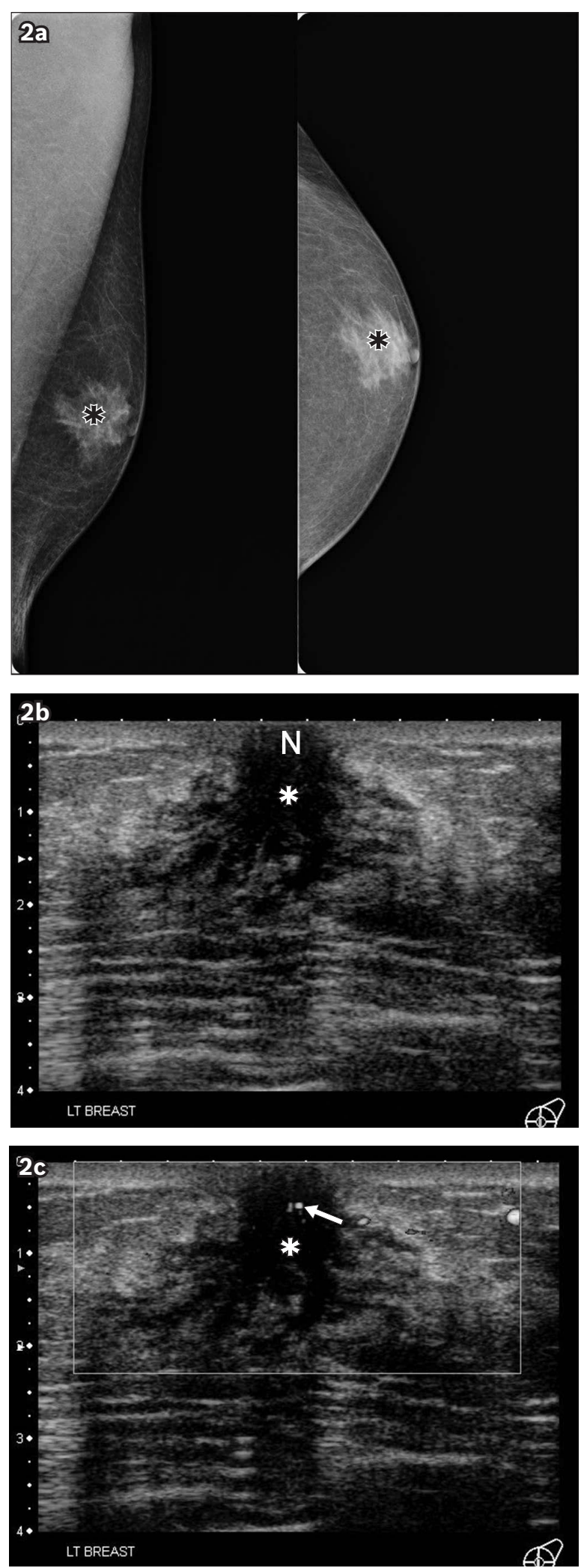

Fig. 2 Nodular gynaecomastia. (a) Mammographic images show a subareolar, fan-shaped density with indistinct borders (black asterisks). (b \& c) Corresponding US images show a fan-shaped subareolar hypoechoic change in echotexture (white asterisks), which is associated with increased internal vascularity (white arrow) that blends into the surrounding hyperechoic fatty tissue.

mimicking a breast malignancy. In order to differentiate gynaecomastia from breast malignancy via imaging, one must recognise the different patterns of gynaecomastia and their corresponding underlying histological changes. The common
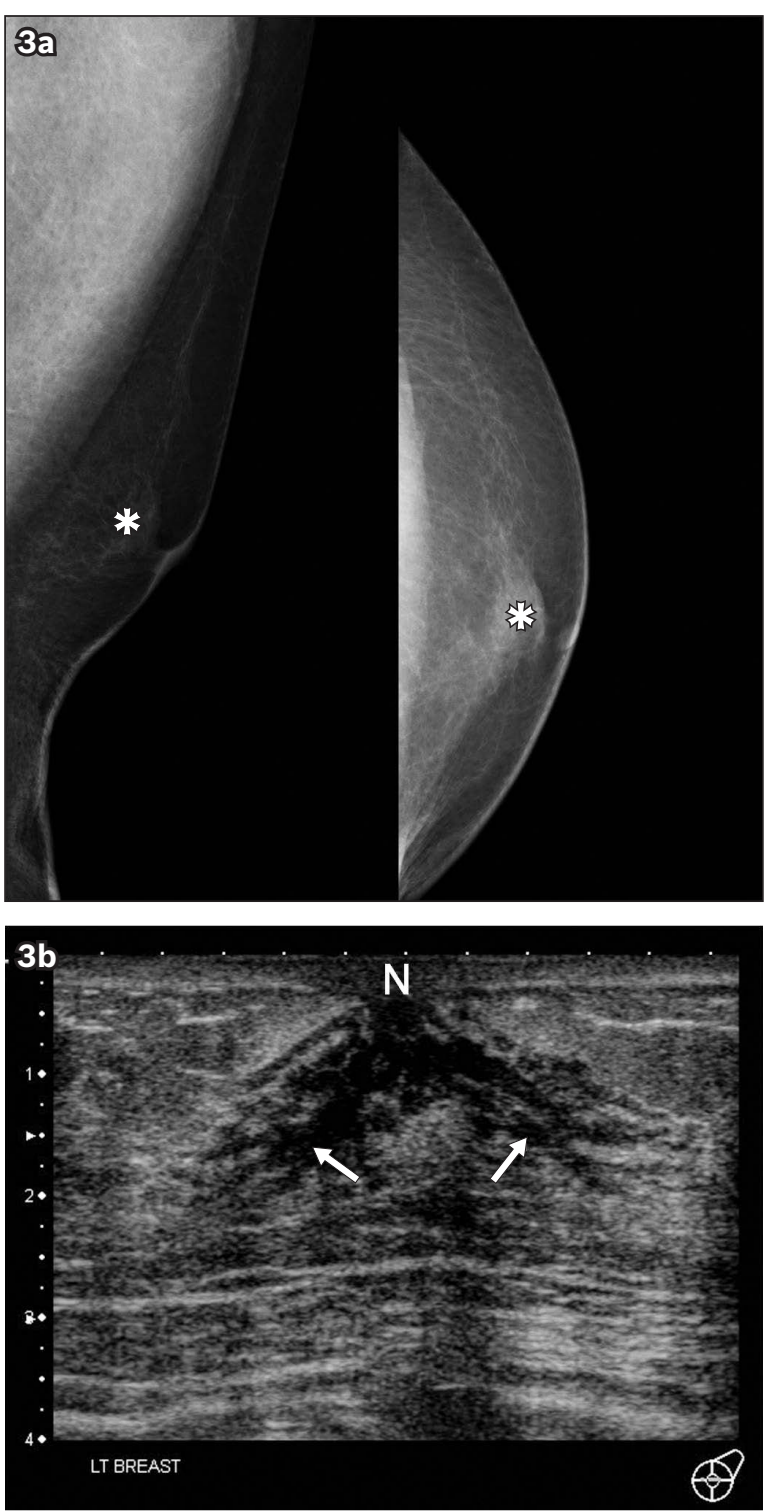

Fig. 3 Dendritic gynaecomastia. (a) Mammographic images show a subareolar density that radiates into the surrounding fatty tissue (white asterisks). (b) US image shows typical hypoechoic 'finger-like' or 'spider-leg type' projections that insinuate into the more echogenic surrounding fibrous breast tissue (white arrows).

categories of gynaecomastia are: (a) nodular type, seen in the early and florid phase of gynaecomastia; (b) dendritic type, seen in the chronic and quiescent phase; and (c) diffuse type, commonly encountered in patients receiving exogenous oestrogen.

The nodular type of gynaecomastia appears as a subareolar, fan-shaped density on mammography and shows indistinct borders (Fig. 2a), as the fibroglandular tissue becomes interspersed with the surrounding fatty tissue. Ultrasonography shows a fan-shaped, subareolar hypoechoic change in echotexture (Fig. 2b), which is related to ductal, epithelial and stromal proliferation, as well as increased internal vascularity (Fig. 2c). The margins appear indistinct, as the gynaecomastia tissue blends into the surrounding fatty tissue, which is relatively more hyperechoic. The nodular pattern of gynaecomastia may be mistaken for malignancy due to its indistinct margins and hypervascularity. ${ }^{(3)}$ However, the indistinct margins are 

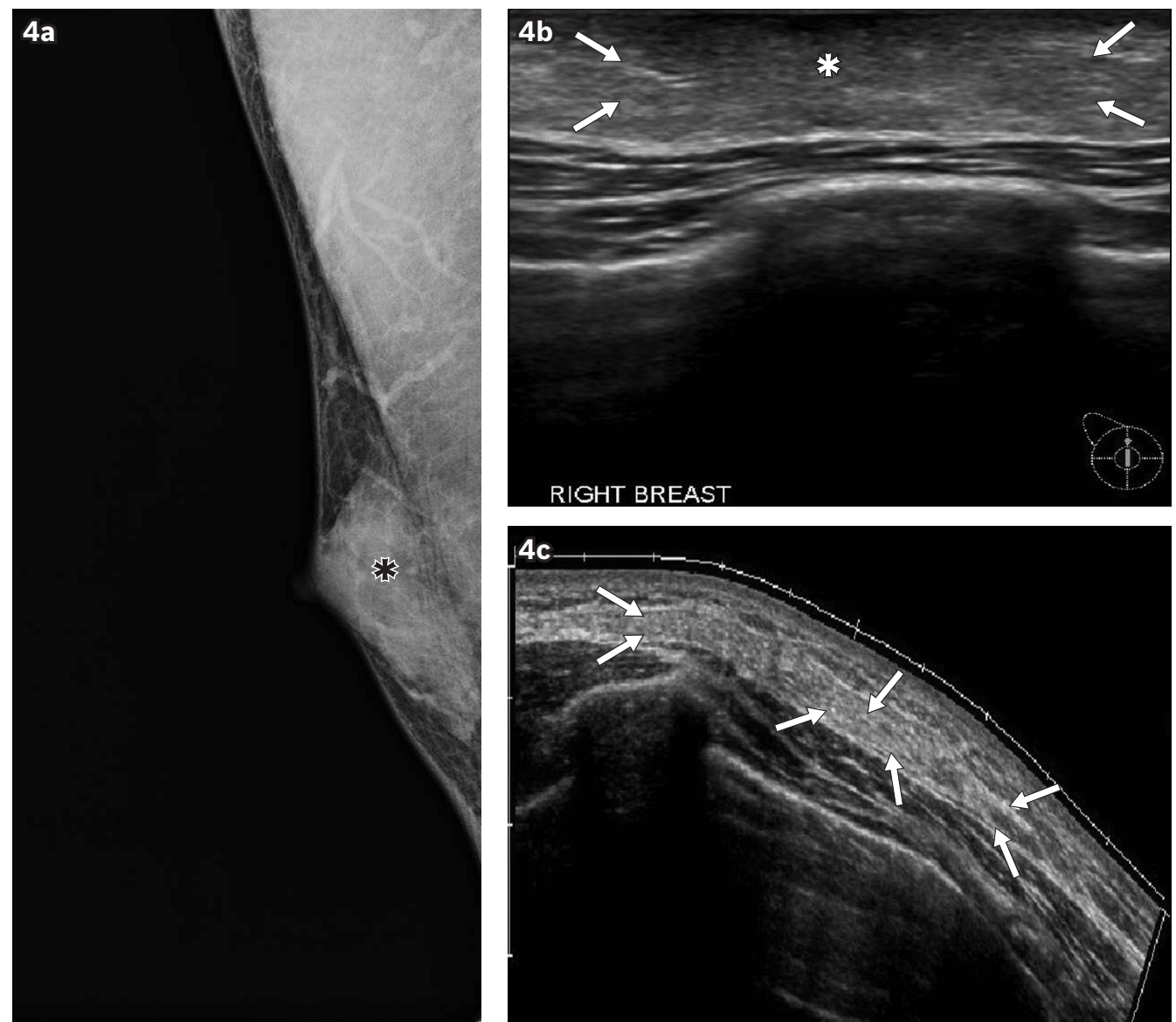

Fig. 4 Diffuse gynaecomastia. (a) Mammographic image shows the appearance of a diffuse gynaecomastia (black asterisk) resembling a heterogeneously dense female breast. ( $b$ \& c) US images show mixed nodular and dendritic features (white asterisk) associated with surrounding diffusely echogenic fibrous breast tissue (white arrows).

recognised as a typical feature of gynaecomastia, while hypervascularity may be explained by stromal proliferation, and as such, these features should not be considered worrisome for malignancy.

In dendritic gynaecomastia, there is a more dominant stromal fibrotic process, and projections of proliferated ductal and epithelial elements into the surrounding fibrous breast tissue are present. When a subareolar density that radiates into the surrounding fatty tissue is demonstrated on mammography, it gives rise to a more 'wispy' appearance (Fig. 3a) as compared to nodular gynaecomastia. Ultrasonography reveals typical hypoechoic 'finger-like' or 'spider-leg type' projections that insinuate into the surrounding more echogenic fibrous breast tissue (Fig. 3b). The mammographic appearance of diffuse gynaecomastia resembles that of a heterogeneously dense female breast (Fig. 4a). Ultrasonography shows mixed nodular and dendritic features associated with surrounding diffusely echogenic fibrous breast tissue (Figs. 4b \& c). Additional features that distinguish gynaecomastia from male breast cancer include the absence of a truly discrete mass, central symmetric location under the nipple of the mammographic density or sonographic change in echotexture, as well as the lack of secondary features of malignancy. ${ }^{(3,7,8)}$

In conclusion, a detailed clinical history and careful physical examination are important prerequisites when evaluating a male patient who presents with a breast mass. The imaging findings from mammography and breast ultrasonography are equally useful adjuncts in identifying cases suspicious for male breast carcinoma that warrant biopsy.

ABSTRACT A 51-year-old man with no significant medical history was referred to our institution for further management of a palpable, painless right breast lump that had been gradually increasing in size for a period of six months. Physical examination revealed a firm right breast lump and bloody right nipple discharge, but no skin involvement or axillary lymphadenopathy was observed. Subsequent mammography and breast ultrasonography demonstrated a discrete, heterogeneous and vascular right breast mass with spiculated and angulated margins. The breast mass was found to be an invasive ductal carcinoma on ultrasonography-guided core needle biopsy. This case illustrates that a combination of detailed clinical history, careful physical examination and radiological assessment using mammography and breast ultrasonography may be used to identify cases suspicious for male breast carcinoma that warrant biopsy.

Keywords: gynaecomastia, male breast carcinoma, mammography, ultrasonography 


\section{REFERENCES}

1. Niewoehner CB, Schorer AE. Gynaecomastia and breast cancer in men. BMJ 2008; 336:709-13.

2. Heller KS, Rosen PP, Schottenfeld D, Ashikari R, Kinne DW. Male breast cancer: a clinicopathologic study of 97 cases. Ann Surg 1978; 188:60-5.

3. Chen L, Chantra PK, Larsen LH, et al. Imaging characteristics of malignant lesions of the male breast. Radiographics 2006; 26:993-1006.

4. Evans GF, Anthony T, Turnage RH, et al. The diagnostic accuracy of mammography in the evaluation of male breast disease. Am J Surg 2001; 181:96-100. Erratum in: Am J Surg 2001; 181:579.

5. Adibelli ZH, Oztekin O, Postaci H, Uslu A. The Diagnostic Accuracy of Mammography and Ultrasound in the Evaluation of Male Breast Disease: A New Algorithm. Breast Care (Basel) 2009; 4:255-9.

6. Patterson SK, Helvie MA, Aziz K, Nees AV. Outcome of men presenting with clinical breast problems: the role of mammography and ultrasound. Breast J 2006; 12:418-23.

7. Muttarak M. Clinics in diagnostic imaging (88). Gynaecomastia of the right breast and carcinoma of the left breast. Singapore Med J 2003; 44:433-7.

8. Gunhan-Bilgen I, Bozkaya H, Ustun E, Memis A. Male breast disease: clinical, mammographic, and ultrasonographic features. Eur J Radiol 2002; 43:246-55.

9. Yang WT, Whitman GJ, Yuen EH, Tse GM, Stelling CB. Sonographic features of primary breast cancer in men. AJR Am J Roentgenol 2001; 176:413-6.

10. Hittmair AP, Lininger RA, Tavassoli FA. Ductal carcinoma in situ (DCIS) in the male breast: a morphologic study of 84 cases of pure DCIS and 30 cases of DCIS associated with invasive carcinoma - a preliminary report. Cancer 1998; 83:2139-49. 


\section{SINGAPORE MEDICAL COUNCIL CATEGORY 3B CME PROGRAMME} (Code SMJ 201306B)

Question 1. With regard to male breast carcinoma:

(a) It accounts for approximately $1 \%$ of all cases of breast carcinoma.

(b) The mean age at presentation is 65 years old.

(c) It may present as a hard, painless mass fixed to the skin or pectoral fascia.

(d) It may present with bloody nipple discharge.

Question 2. Risk factors for male breast carcinoma include:

(a) BRCA1 and BRCA2 breast cancer mutation genes.

(b) Klinefelter's syndrome.

(c) Hyperoestrogenism.

(d) Prior chest wall irradiation.

Question 3. Concerning male breast carcinoma:

(a) The majority of cases comprise moderate or poorly differentiated invasive ductal carcinoma (IDC).

(b) The incidence of ductal carcinoma in situ (DCIS) without associated IDC is rare.

(c) Microcalcifications appear coarser and are less frequently rod-shaped when compared to female breast carcinoma.

(d) It should be suspected if a discrete subareolar mass that is eccentrically located to the nipple is found on imaging.

Question 4. Regarding gynaecomastia:

(a) It is characterised by hyperplasia of the glandular elements of the male breast.

(b) It usually occurs in pre-pubertal boys.

(c) It may coexist with male breast carcinoma in up to $40 \%$ of cases.

(d) It is associated with increased serum oestradiol to testosterone ratio.

Question 5. Regarding gynaecomastia:

(a) On mammography, a central symmetrical subareolar wedge or fan-shaped density is seen in nodular gynaecomastia.

(b) On ultrasonography, hypoechoic 'finger-like' projections into the surrounding echogenic fibrous stroma are seen in dendritic gynaecomastia.

(c) The appearance of diffuse gynaecomastia is similar to that of a heterogeneously dense female breast.

(d) Gynaecomastia may appear mass-like with poorly marginated borders on mammography and ultrasonography, mimicking a breast malignancy.

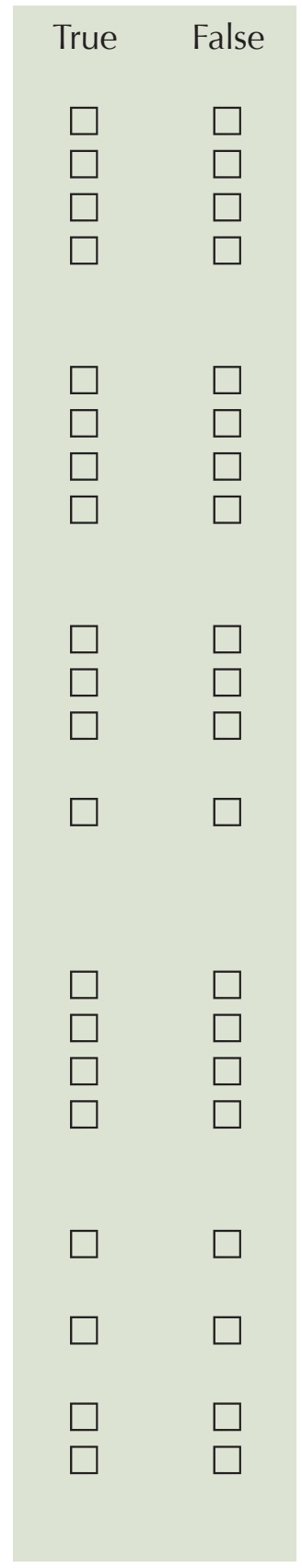

\footnotetext{
Doctor's particulars:

Name in full

MCR number

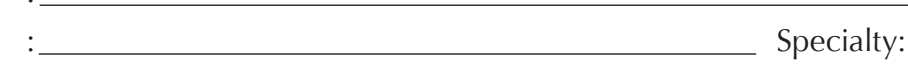

Email address

(1) Log on at the SMJ website: http://www.sma.org.sg/publications/smjcurrentissue.aspx and select the appropriate set of questions. (2) Provide your name, email address and MCR number. (3) Select your answers and click "Submit".

RESULTS:

(1) Answers will be published in the SMJ August 2013 issue. (2) The MCR numbers of successful candidates will be posted online at the SMJ website by 29 July 2013.

(3) Passing mark is $60 \%$. No mark will be deducted for incorrect answers. (4) The SMJ editorial office will submit the list of successful candidates to the Singapore

Medical Council. (5) One CME point is awarded for successful candidates.

Deadline for submission: (June 2013 SMJ 3B CME programme): 12 noon, 23 July 2013.
} 